\title{
Realising the decomposition of a multi- frequency signal under the coloured noise background by the adaptive stochastic resonance in the non-linear system with periodic potential
}

ISSN 1751-9675

Received on 9th November 2017

Revised 15th March 2018

Accepted on 4th April 2018

E-First on 5th June 2018

doi: 10.1049/iet-spr.2017.0532

www.ietdl.org

\author{
Xiaogang Huang1,2, Jingling Zhang ${ }^{3}$, Meilei Lv², Gang Shen³, Jianhua Yang ${ }^{3,4}$ 凶 \\ ${ }^{1}$ Department of Electrical Automation, Shanghai Maritime University, Shanghai 201306, People's Republic of China \\ ${ }^{2}$ College of Electrical and Information Engineering, Quzhou University, Quzhou 324000, People's Republic of China \\ ${ }^{3}$ School of Mechatronic Engineering, China University of Mining and Technology, Xuzhou 221116, People's Republic of China \\ ${ }^{4}$ Department of Mechanical Engineering, University of Michigan, Ann Arbor, MI 48109, USA \\ 凶-mail: jianhuayang@cumt.edu.cn
}

\begin{abstract}
The authors investigate a multi-frequency signal which is decomposed failure by the traditional empirical mode decomposition (EMD) method. Moreover, the multi-frequency signal submerged in the coloured noise increases the difficulty in signal decomposition. As a result, this noisy signal is decomposed unsuccessfully by the cooperation of the adaptive stochastic resonance (SR) in the classic bistable system and EMD. Then, a method combined adaptive SR in the periodic potential system and EMD is put forward to realise the decomposition. Meanwhile, the random particle swarm optimisation algorithm is applied to reach the optimal situation when signal-to-noise ratio attains the maximum value. Different simulation results verify the effectiveness of the proposed method. The proposed method might be useful in dealing with signal processing problems.
\end{abstract}

\section{Introduction}

The method of empirical mode decomposition (EMD) was first reported in 1998 [1]. This method is different from traditional signal processing methods because it decomposes a complicated signal into limited intrinsic mode functions (IMFs). It is suitable for processing non-linear and non-stationary signals.

Although the EMD method solves some complicated signals successfully [2-5], it still has difficulty when the signal submerged in strong noise background. This problem may be solved by the cooperation of stochastic resonance (SR) and EMD [6,7]. The theory of SR was firstly put forward by Benzi et al. [8] and has been widely used in signal processing fields [9]. At present, the classical model of SR is usually a bistable system [10-12]. In fact, SR can be presented in a variety of non-linear systems. For example, the periodic potential system is one kind of typical nonlinear system [13-15]. Further, the method of SR in periodic system has superiority in improving the signal-to-noise ratio (SNR) $[16,17]$. Nowadays, the method of SR in bistable system is studied widely. However, the method of SR in the periodic potential system is still needed to be investigated intensively, especially in the engineering applications.

In most studies, the Gaussian white noise is usually added in signals to simulate a noisy background [18]. However, the noise of the realistic model may be in the coloured noise form [19-25]. Therefore, it is necessary to research the signal under coloured noise background. Fang et al. [26] explored the effects of the coloured noise on the resonance at the subharmonic frequency in bistable systems. Xu et al. [27] proposed the effects of coloured noise on multi-frequency signal processing via SR with tuning system parameters. Ma et al. [28] researched the coherence resonance induced by coloured noise near the Hopf bifurcation. However, all the aforementioned references have not solved the EMD problem when the multi-frequency signal submerged in the coloured noise background.

The remaining of this paper is organised as follows. In Section 2, we will introduce the theory of EMD and the model of coloured noise. In Section 3, we will show the considered multi-frequency signal which cannot be decomposed by EMD directly under the coloured noise background. In Section 4, the method of adaptive $\mathrm{SR}$ in the classic bistable system and the method of adaptive SR in the periodic potential system are introduced. Then, two kinds of adaptive SR are combined with EMD respectively to process the multi-frequency signal under the coloured noise background. Finally, the main conclusions of the paper are given in Section 5.

\section{Theory and model}

\subsection{Theory of EMD}

The method of EMD decomposes a multi-frequency signal into the following form:

$$
x(t)=\sum_{i=1}^{n} c_{i}(t)+r_{n}(t)
$$

In (1), $c_{i}$ is the $i$ th IMF component. $r_{n}(t)$ is the residual amount after the IMFs are extracted. The purpose of EMD is to extract the components of the signal from high frequency to low frequency. That is, the components of the highest frequencies will be firstly got, then the second high frequencies, and ultimately residual components with frequencies close to zero. The termination condition of the decomposition is that $r_{n}(t)$ becomes a monotonic function or a constant. Then the high frequency components are the most important components as they always represent the main characteristics of the signal. The residual amount stands for the average trend of the signal. However, the decomposed data is usually complex and cannot predict how many IMF components are included in practical applications. Therefore, the termination condition of decomposition is subjectively determined by the researcher according to the research needs. Herein, we subjectively determine the number of IMFs because the noisy signal is complicated. Through analysing each IMF, we get the features of data information at different time scales. In addition, the EMD method is based on the following three assumptions. First, the signal must have at least two extreme points which are one 


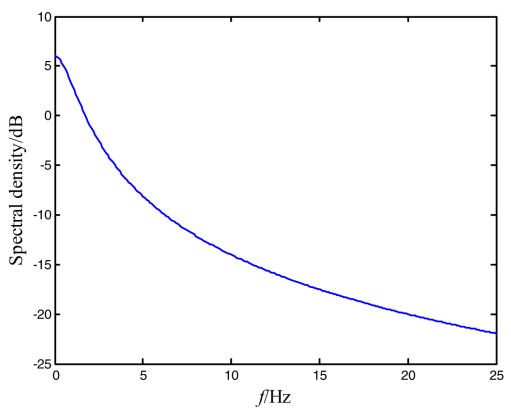

Fig. 1 Power spectrum of the exponential coloured Gaussian noise
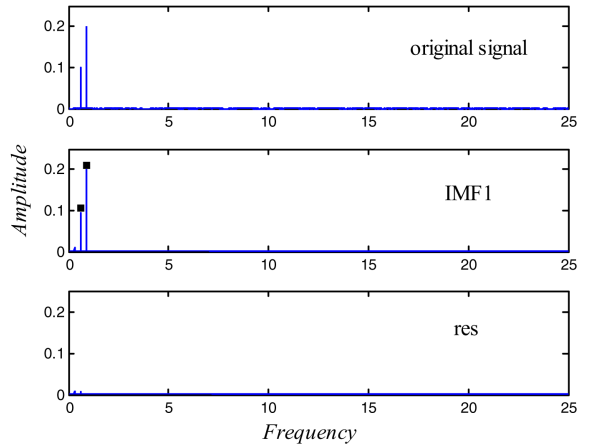

Fig. 2 Frequency spectrum of the two-frequency signal is decomposed by $E M D$

maximum point and one minimum point. Second, the characteristic time scale is defined as the time interval between adjacent extremum points. Finally, if the signal owns no extreme points but only inflection points, it should be differential one or more times to obtain extreme points and then integrating the results to get the corresponding components [29].

\subsection{Model of coloured noise}

The exponential coloured Gaussian noise is widely used in engineering filed. It can be modelled by the Ornstein-Uhlenbeck process

$$
\frac{\mathrm{d} n(t)}{\mathrm{d} t}=-\frac{n(t)}{\tau_{c}}+\frac{\Gamma(t)}{\tau_{c}},
$$

where $\tau_{c}$ is the correlation time of the noise and $\Gamma(t)$ is an ideal white noise. The statistic characteristics of $n(t)$ are

$$
\langle n(t)\rangle=0, \quad\left\langle n(t) n\left(t^{\prime}\right)\right\rangle=\frac{D}{\tau_{c}} \mathrm{e}^{-\left|t-t^{\prime}\right| / \tau_{c}},
$$

where $D$ stands for the noise intensity. When the value of $\tau_{c}$ tends to zero, the coloured noise will approach to the Gaussian white noise. The power spectrum of the exponential coloured Gaussian noise is expressed as

$$
S(f)=\frac{2 D}{1+\left(\tau_{c} f\right)^{2}},
$$

where the relationship between $S(f)$ and $f$ is the Lorenz function. When $D$ and $\tau_{c}$ are set as 2 and 1, respectively, in (4), Fig. 1 reveals the power spectrum of the exponential coloured Gaussian noise. The amplitude of the coloured noise is gradually reduced from zero and the energy in high frequency is insignificant.

\section{Multi-frequency signal}

We use a two-frequency signal and a three-frequency signal to explain the proposed method.

The two-frequency signal is described by

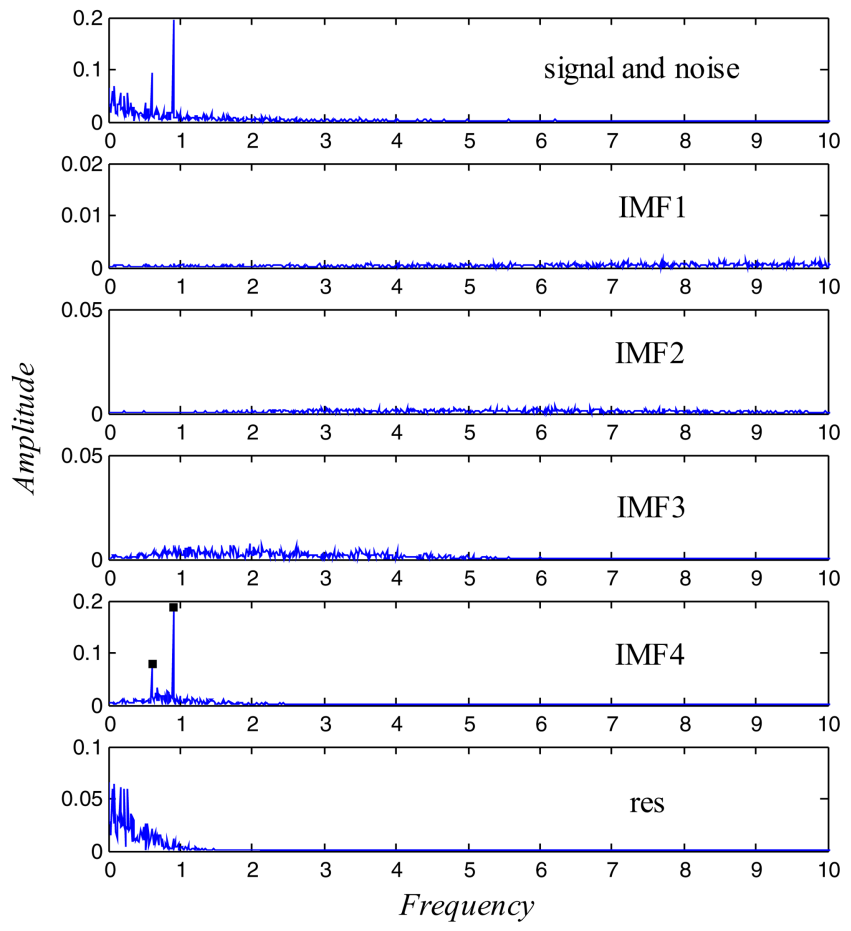

Fig. 3 Frequency spectrum of the two-frequency signal is decomposed by EMD under the coloured noise background. In IMF4, the highest points are located at 0.6 and $0.9 \mathrm{~Hz}$

$$
s_{1}(t)=A_{1} \cos \left(2 \pi f_{1} t\right)+A_{2} \sin \left(2 \pi f_{2} t\right) .
$$

In (5), we let $A_{1}=0.1, A_{2}=0.2, f_{1}=0.6, f_{2}=0.9$.

The three-frequency signal is described by

$$
s_{2}(t)=B_{1} \cos \left(2 \pi F_{1} t\right)+B_{2} \sin \left(2 \pi F_{2} t\right)+B_{3} \cos \left(2 \pi F_{3} t\right) .
$$

In (6), we let $B_{1}=0.1, B_{2}=0.1, B_{3}=0.1, F_{1}=0.2, F_{2}=0.5, F_{3}=$ 0.8 .

First, the two-frequency signal is decomposed by EMD directly. Fig. 2 shows the decomposition results. In the model of the coloured noise and in the process of EMD, without special remarks, we always set $\mathrm{d} t=0.02, \tau_{c}=1, D=2$ and $f_{\mathrm{s}}=50, N=$ 4000. IMF1 is almost the original signal and two frequency components do not be decomposed into different IMFs. Fig. 3 describes the frequency spectrums of the noisy two-frequency signal which is processed by EMD directly. In Fig. 3, we find the highest points appearing in IMF4 are located at 0.6 and $0.9 \mathrm{~Hz}$. Mode mixing phenomenon consists of two types. One is that a single IMF contains different frequencies and the other is that different IMFs include the same frequency. As a result, the signals in Figs. 2 and 3 are all show mode mixing phenomenon and not in successful decomposition.

Fig. 4 shows the frequency spectrum of the noisy threefrequency signal which is processed by EMD directly. In Fig. 4, the highest point in IMF4 is located at $0.8 \mathrm{~Hz}$. The two highest points in IMF5 are located at 0.5 and $0.8 \mathrm{~Hz}$, respectively. In IMF6, the highest point is located at $0.2 \mathrm{~Hz}$. The frequency of $0.8 \mathrm{~Hz}$ appears in IMF4 and IMF5. The frequencies of 0.5 and $0.8 \mathrm{~Hz}$ both exist in IMF5. It is a mode mixing phenomenon and the noisy signal decomposed failure.

\section{Method of EMD combined with the adaptive SR}

The traditional SR makes the non-linear system achieve its best output by optimising the noise intensity. However, it has some shortness because sometimes the noise cannot be reduced in some engineering situations. So, the method of adaptive SR in which the noise intensity is fixed and the system parameters are adjusted was proposed to overcome the shortness [30]. 32]

The model of SR is expressed by the Langevin equation [31, 


$$
\frac{\mathrm{d} x}{\mathrm{~d} t}=-U^{\prime}(x)+s(t)+n(t)
$$

where $U(x)$ is the potential function, $s(t)$ stands for the multifrequency signal and $n(t)$ represents the coloured noise in this paper.

In most cases, the SR in traditional bistable system is widely investigated. The bistable potential function is

$$
U(x)=-\frac{a}{2} x^{2}+\frac{b}{4} x^{4}
$$

where $a>0$ and $b>0$ are the system parameters. Substituting (8) into (7), then (7) is simplified to

$$
\frac{\mathrm{d} x}{\mathrm{~d} t}=a x-b x^{3}+s(t)+n(t)
$$

Equation (9) is the model of SR in bistable system and we call this kind of SR as BSR for simplify.

Besides the bistable potential, the periodic function is another form of non-linear potential, which is given as

$$
U(x)=-a \cos (b x),
$$

where $a>0$ and $b>0$ are the system parameters. Substituting (10) into (7), one obtains

$$
\frac{\mathrm{d} x}{\mathrm{~d} t}=-a b \sin (b x)+s(t)+n(t) .
$$

Equation (11) is the model of SR in periodic potential system and this type of equation can be solved by the fourth-order RungeKutta algorithm [33]. We call the SR in periodic potential system as PSR for simplify.

In this paper, in order to realise the adaptive SR and improve the computation efficiency, the random particle swarm optimisation (RPSO) algorithm [34-36] is applied to obtain the optimal parameters $a$ and $b$. Meanwhile, SNR is set as the evaluation index in the calculation [37]. SNR is defined as

$$
\mathrm{SNR}=10 \log _{10}\left(\frac{S(f)}{N(f)}\right)
$$

where $S(f)$ is the energy at the signal frequency $f$ and $N(f)$ is the average energy of the noise background at the signal frequency neighbourhood. $S(f)$ and $N(f)$ are, respectively, discretised and calculated by

$$
\left\{\begin{array}{l}
S(f)=|X(k)|^{2} \\
N(f)=\frac{1}{2 M} \sum_{j=1}^{M}\left(|X(k-j)|^{2}+|X(k+j)|^{2}\right)
\end{array},\right.
$$

where $X(\cdot)$ is the amplitude of the response and $X(k)$ is the amplitude at the signal frequency $f$. To calculate the noise energy around $f$, we consider the average noise energy in the discrete amplitude spectrum in the interval $f-M \Delta f$ and $k+M \Delta f$, where $\Delta f$ is the step of the discrete amplitude spectrum. For discrete calculation, it is carried out by (13). Actually, the SNR here is the local SNR because the choice of $M$ and $N(f)$ represents the average noise energy around the signal frequency. In the following calculations, $M$ is set as 10 .

The main steps of the RPSO algorithm are as follows:

i. Set the initialisation condition. The learning factors are usually set as 2. The maximum and minimum values of the mean random weights are set as 0.8 and 0.5 , respectively. The variance of the mean random weights is set as 0.2 . These above values are generally applicable to the general situation. The appropriate values of initialised groups number and the iterations number are selected according to the effectiveness and speed of the operation. Herein, the number of initialised groups is 40 and the number of iterations is 50 . Moreover, the space dimension is set as two because two system parameters are needed to be optimised.

ii. Initialise the individual particles in the population. Randomly initialise the particle's position and velocity.

iii. Calculate the fitness of each particle and find the local optimum and global optimum.

iv. Enter the main loop. First, update the position and velocity of the particle. Second, recalculate the fitness of each particle and update the local optimum and global optimum. Third, judge whether the optimal value between 0 and 2 and whether the maximum number of iterations has been reached. If not, continue the loop. If reached, go to the next step.

v. The best result will be obtained by the above steps.

Fig. 5 shows the concrete steps of the signal processing based on the combination of adaptive SR and EMD. According to Fig. 5, these noisy multi-frequency signals which have been processed by adaptive BSR or adaptive PSR will be decomposed by EMD.

The two-frequency signal under coloured noise background is firstly processed. Fig. 6 depicts the frequency spectrums of the combination of adaptive BSR or adaptive PSR with EMD which are optimised at $0.6 \mathrm{~Hz}$. In Fig. $6 a$, the highest point is located at $0.9 \mathrm{~Hz}$ in IMF2. In IMF3, the highest points are located at 0.6 and $0.9 \mathrm{~Hz}$. Unfortunately, the mode mixing problem is not solved completely and the failure decomposition still exists. In Fig. 6b, 0.9 and $0.6 \mathrm{~Hz}$ appear in IMF2 and IMF3, respectively. In addition, there is also a highest point in the IMF4. It is the subharmonic frequency or the combined frequency [38]. Different from the situation in bistable system, it is a successful decomposition because different frequencies appear in different IMFs. The reason for the same signal decomposed failure after BSR and decomposed successful after PSR is that optimisation effect of PSR is better than BSR. The method of PSR equivalently optimises an infinite number of terms because sinx in the model of PSR includes an infinite number of terms when it expands into Taylor series. However, the method of BSR only optimises two terms. Hence, the

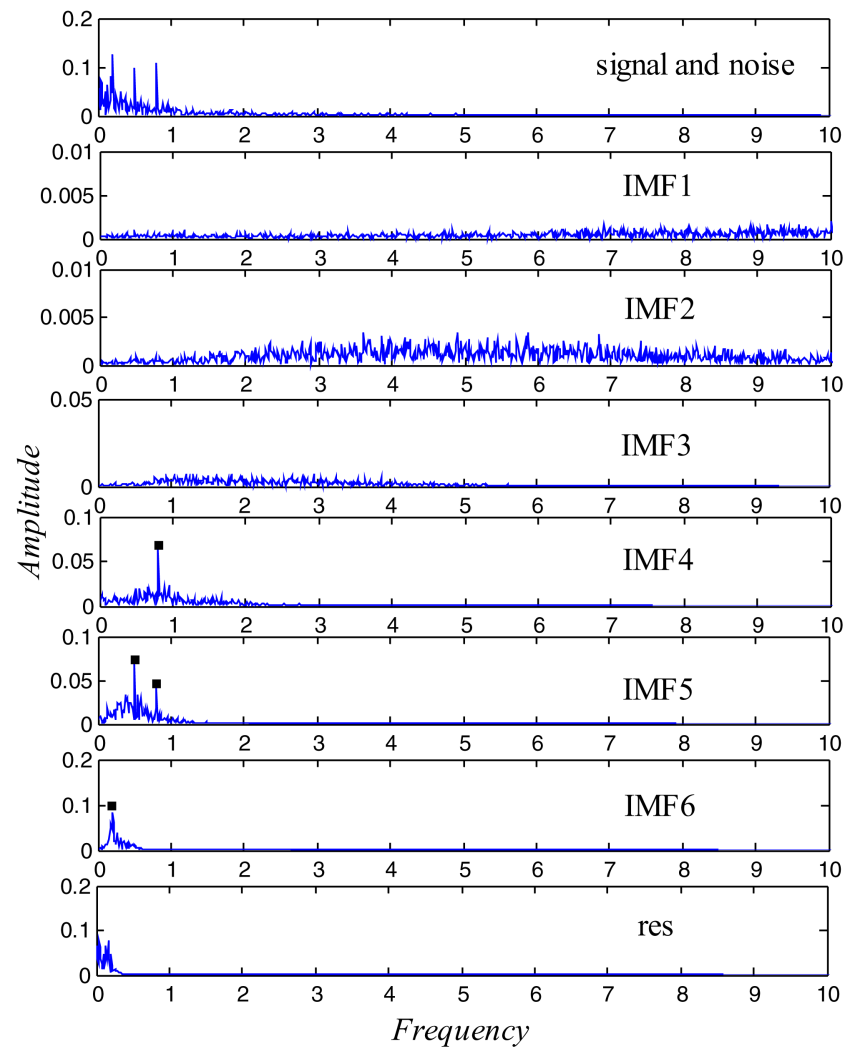

Fig. 4 Frequency spectrum of the three-frequency signal is decomposed by EMD under the coloured noise background. In IMF4, the highest point is located at $0.8 \mathrm{~Hz}$. In IMF5, the highest points are located at 0.5 and $0.8 \mathrm{~Hz}$. In IMF6, the highest point is located at $0.2 \mathrm{~Hz}$ 


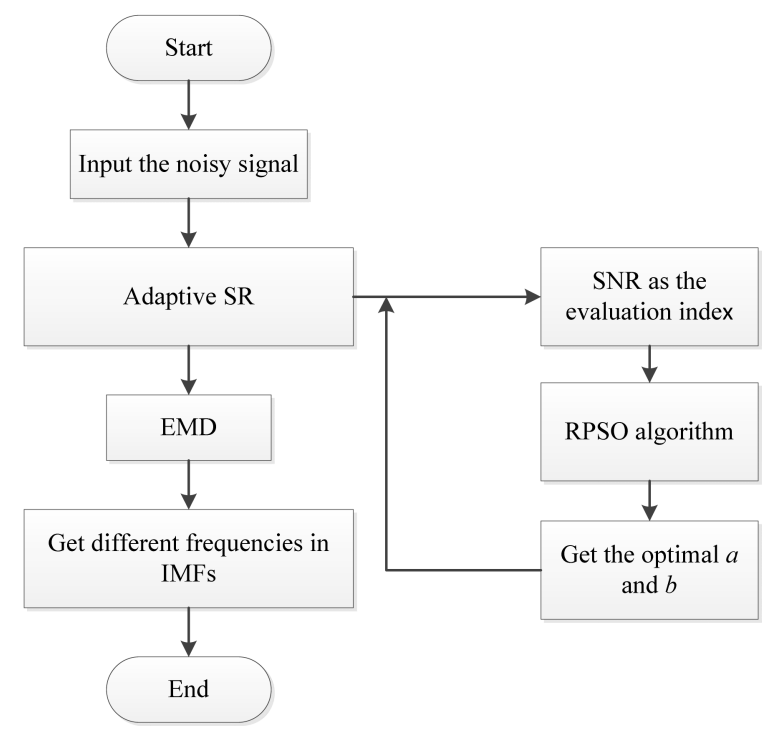

Fig. 5 Flowchart of the combination of the adaptive SR and EMD
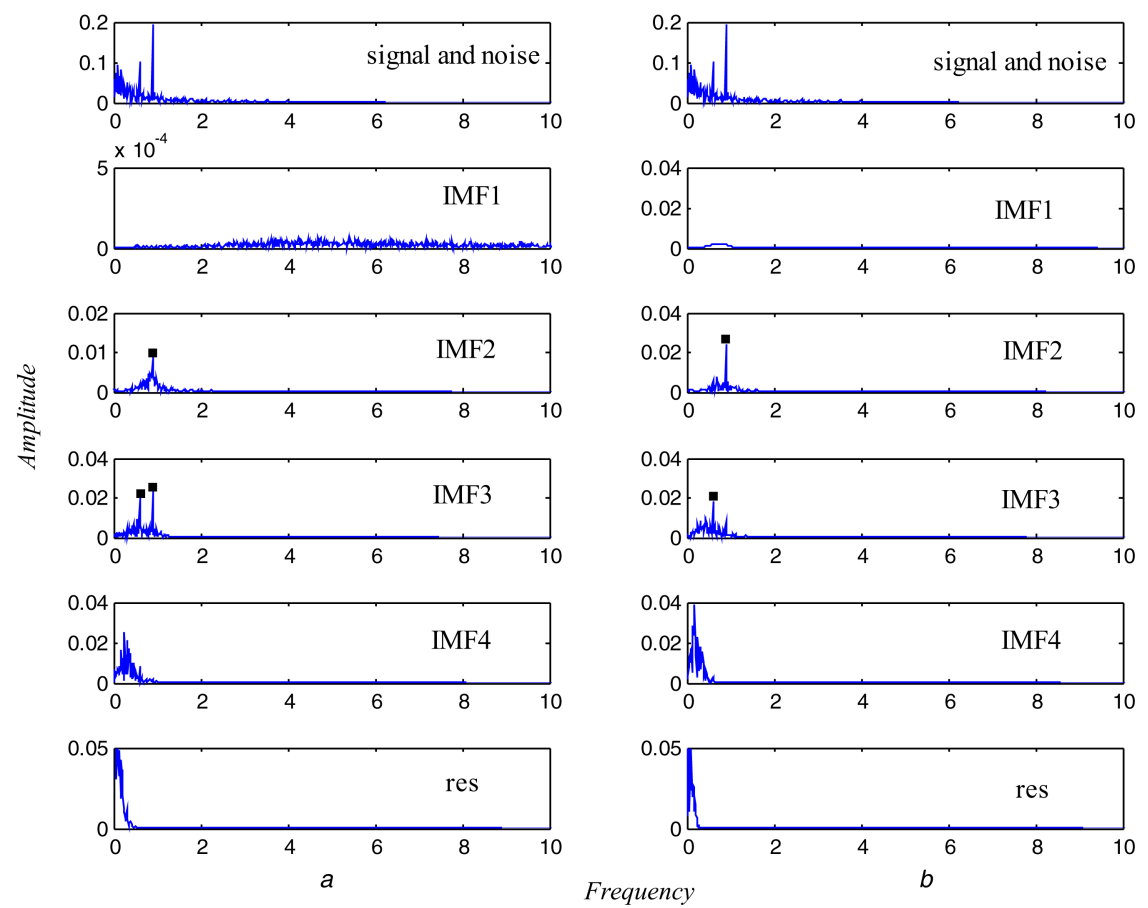

Fig. 6 Frequency spectrums of the noisy two-frequency signals are decomposed by EMD after adaptive BSR or adaptive PSR at $0.6 \mathrm{~Hz}$, when the noise intensity is set as 2

(a) Frequency spectrum of the noisy two-frequency signal is decomposed by EMD after adaptive BSR at $0.6 \mathrm{~Hz}$ : optimal output with $a=0.128$ and $b=1.175$. In IMF2, the highest point is located at $0.9 \mathrm{~Hz}$. In IMF3, the highest points are located at 0.6 and $0.9 \mathrm{~Hz}$, (b) Frequency spectrum of the noisy two-frequency signal is decomposed by EMD after adaptive PSR at $0.6 \mathrm{~Hz}$ : optimal output with $a=1.032$ and $b=0.6523$. In IMF2, the highest point is located at $0.9 \mathrm{~Hz}$. In IMF3, the highest point is located at $0.6 \mathrm{~Hz}$

method of PSR has better effect and then can reach better decomposition condition. Fig. 7 depicts the frequency spectrums of the combination of adaptive BSR or adaptive PSR with EMD which is optimised at $0.9 \mathrm{~Hz}$. Fig. $7 a$ reveals the same phenomenon as that in Fig. $6 a$. In Fig. $7 b, 0.9$ and $0.6 \mathrm{~Hz}$ appear in IMF2 and IMF3, respectively. Similar to the fact in Fig. $6 b$, it is also a successful decomposition.

Sometimes, the analysis of a single time series is also meaningful because we need to analyse a single time series in engineering problems. However, for the purpose of increasing the conviction of the proposed method, we also use the statistical method to analyse the noisy signal and every IMF is averaged 30 times. Then the sensitivity and robustness of this method are both owned through the analysis of a single time series and statistical series. This two-frequency signal under coloured noise background is optimised at $0.9 \mathrm{~Hz}$ by adaptive BSR or adaptive PSR with EMD. Fig. 8 shows the frequency spectrums by these two methods.
Comparing with Fig. 7, the results in Fig. 8 are in accordance with those in Fig. 7. Therefore, the statistical analysis also verifies the proposed method is valid.

In all the above situations, the intensity of coloured noise is all set as 2 . In order to prove the dependence of the proposed method on the noise intensity in most common situations, two-frequency signals with coloured noise intensities of 8 and 9 are processed. Fig. $9 a$ depicts this two-frequency signal with coloured noise intensity of 8 is handled by the combination of adaptive PSR with EMD. Fig. $9 b$ depicts this two-frequency signal with coloured noise intensity of 9 is handled by the combination of adaptive PSR with EMD. In Fig. 9a, a successful decomposition is shown. Make comparison with Figs. $6 b$ and $9 a$, they reveal the same results. So the validity of the method is not affected by the normal noise intensity in common cases. In Fig. 9b, the interference components cover the signal frequency. Hence, the proposed method is a failure when the noise intensity is more than 8 . However, this noise 

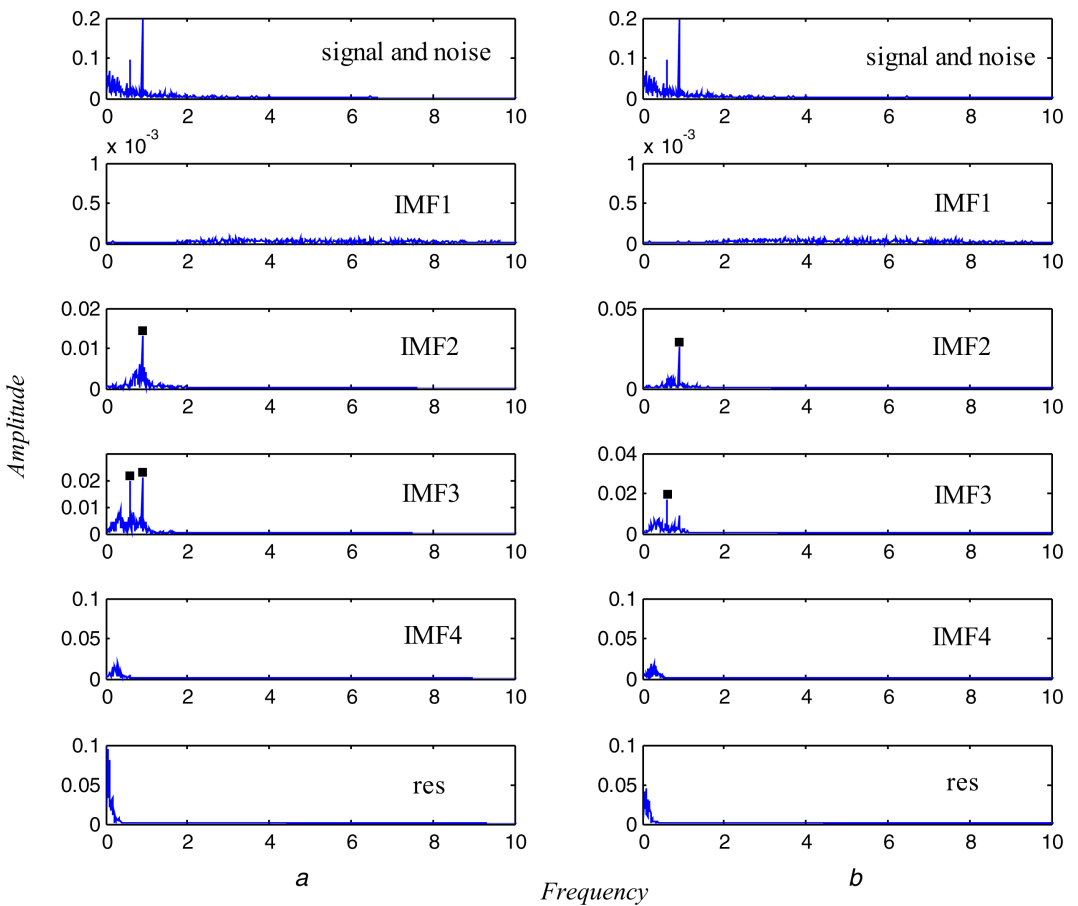

Fig. 7 Frequency spectrums of the noisy two-frequency signals are decomposed by EMD after adaptive BSR or adaptive PSR at 0.9 Hz, when the noise intensity is set as 2

(a) Frequency spectrum of the noisy two-frequency signal is decomposed by EMD after adaptive BSR at $0.9 \mathrm{~Hz}$ : optimal output with $a=0.067$ and $b=0.699$. In IMF2, the highest point is located at $0.9 \mathrm{~Hz}$. In IMF3, the highest points are located at 0.6 and $0.9 \mathrm{~Hz}$, (b) Frequency spectrum of the noisy two-frequency signal is decomposed by EMD after adaptive PSR at $0.9 \mathrm{~Hz}$ : optimal output with $a=0.279$ and $b=1.536$. In IMF2, the highest point is located at $0.9 \mathrm{~Hz}$. In IMF3, the highest point is located at $0.6 \mathrm{~Hz}$
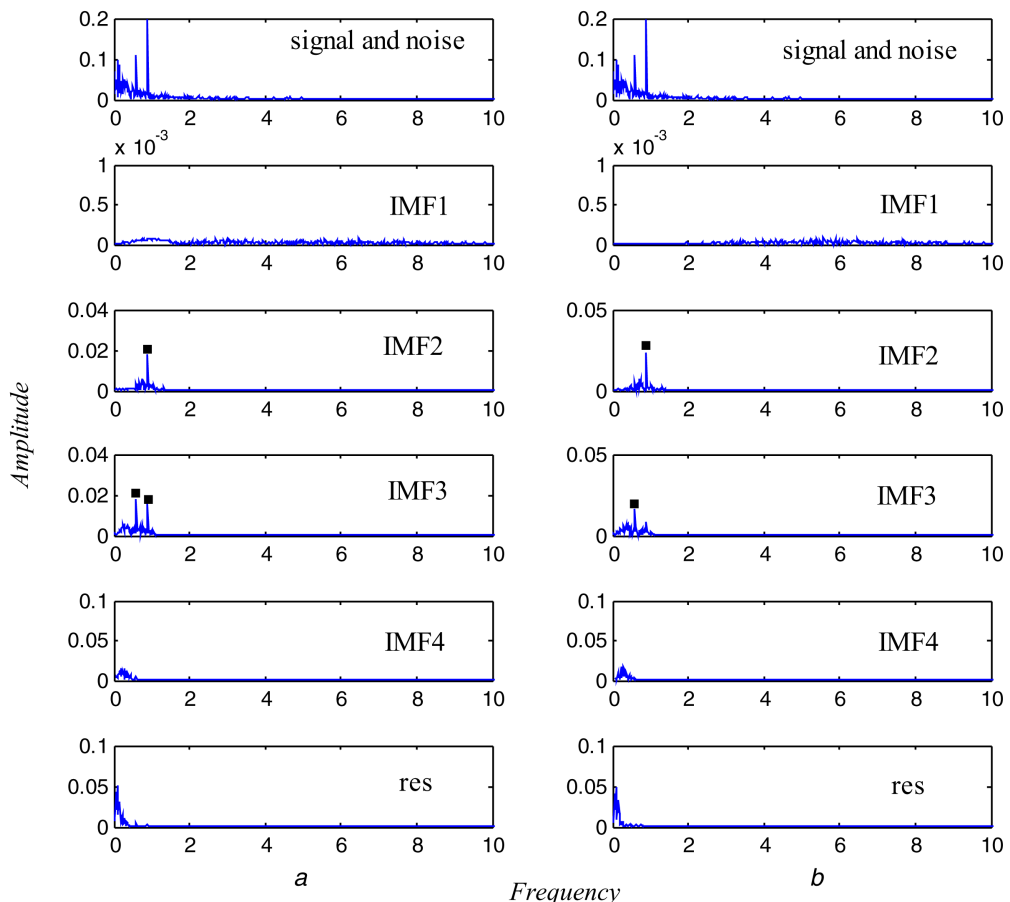

Fig. 8 Frequency spectrums of the noisy two-frequency signals are decomposed with statistical method by EMD after adaptive BSR or adaptive PSR at 0.9 $\mathrm{Hz}$, when the noise intensity is set as 2

(a) Frequency spectrum of the noisy two-frequency signal is decomposed by EMD after adaptive BSR at $0.9 \mathrm{~Hz}$ : every IMF is averaged 30 times. In IMF2, the highest point is located at $0.9 \mathrm{~Hz}$. In IMF3, the highest points are located at 0.6 and $0.9 \mathrm{~Hz}$, (b) Frequency spectrum of the noisy two-frequency signal is decomposed by EMD after adaptive PSR at $0.9 \mathrm{~Hz}$ : every IMF is averaged 30 times. In IMF2, the highest point is located at $0.9 \mathrm{~Hz}$. In IMF3, the highest point is located at $0.6 \mathrm{~Hz}$

intensity range, which suitable for this method can meet most of the actual engineering conditions. Moreover, we can estimate whether this proposed method is suitable for an unknown mixed noisy signal. The variance of this mixed noisy signal is 0.1924 when its noise intensity is 8 . So if the variance of an unknown mixed noisy signal is larger than 0.1924 , the proposed method is not applicable here.
Fig. 10 demonstrates the three-frequency signal under coloured noise background is processed by the cooperation of adaptive BSR or adaptive PSR with EMD. Fig. $10 a$ shows the frequency spectrums of the combination of adaptive BSR and EMD, which are optimised at $0.8 \mathrm{~Hz}$. In Fig. 10a, different frequencies appear in one IMF because the two highest points in IMF3 are 0.5 and 0.8 $\mathrm{Hz}$. Meanwhile, the same frequency appears in the different IMFs as the highest points in IMF4 and IMF5 are all $0.2 \mathrm{~Hz}$. In summary, 

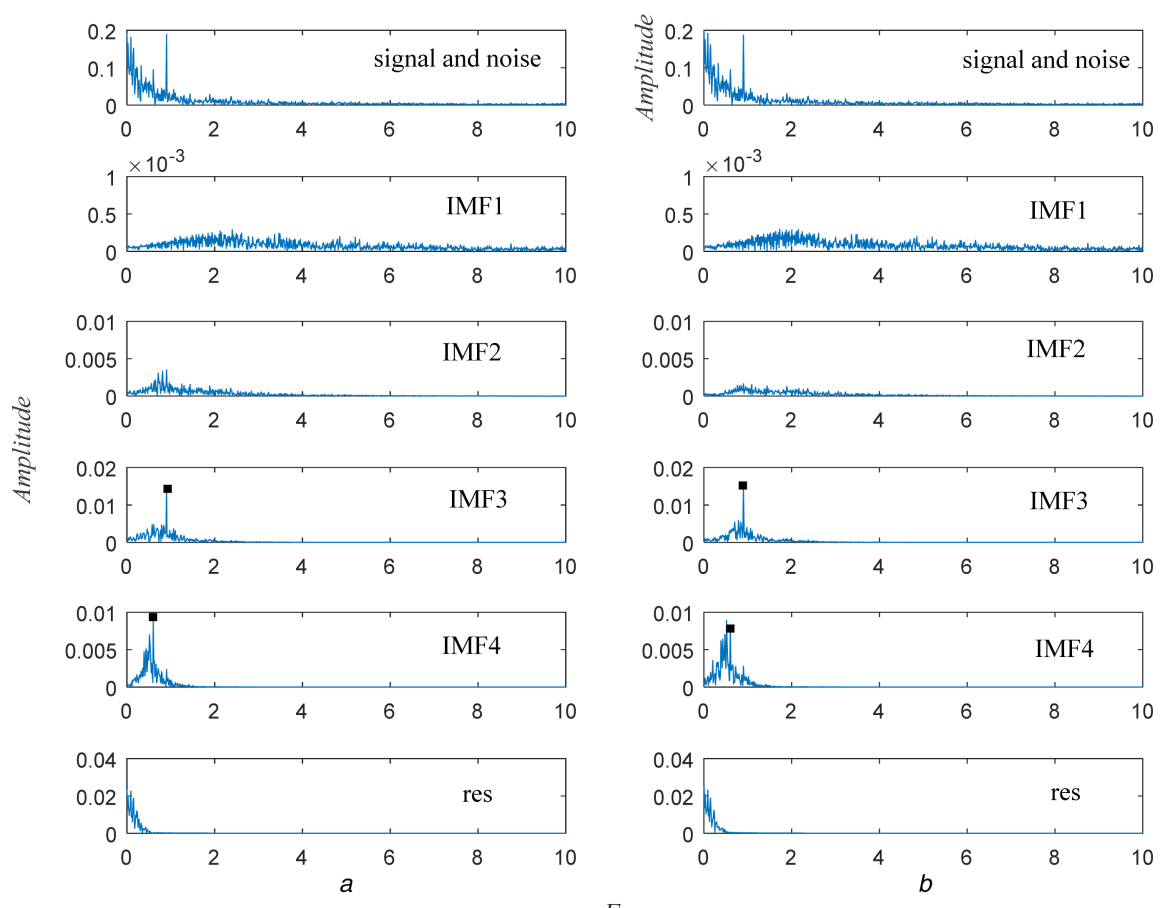

Frequency

Fig. 9 Frequency spectrums of the noisy two-frequency signals are decomposed by EMD after adaptive PSR at $0.6 \mathrm{~Hz}$ when the noise intensities are set as 8 and 9

(a) Frequency spectrum of the noisy two-frequency signal is decomposed by EMD after adaptive PSR at $0.6 \mathrm{~Hz}$ when the noise intensity is 8 : optimal output with $a=1.947$ and $b=$ 1.9883. In IMF3, the highest point is located at $0.9 \mathrm{~Hz}$. In IMF4, the highest point is located at $0.6 \mathrm{~Hz}$, (b) Frequency spectrum of the noisy two-frequency signal is decomposed by EMD after adaptive PSR at $0.6 \mathrm{~Hz}$ when the noise intensity is 9: optimal output with $a=1.9861$ and $b=1.9822$. In IMF3, the highest point is located at $0.9 \mathrm{~Hz}$. In IMF4, the highest point is located at $0.5 \mathrm{~Hz}$
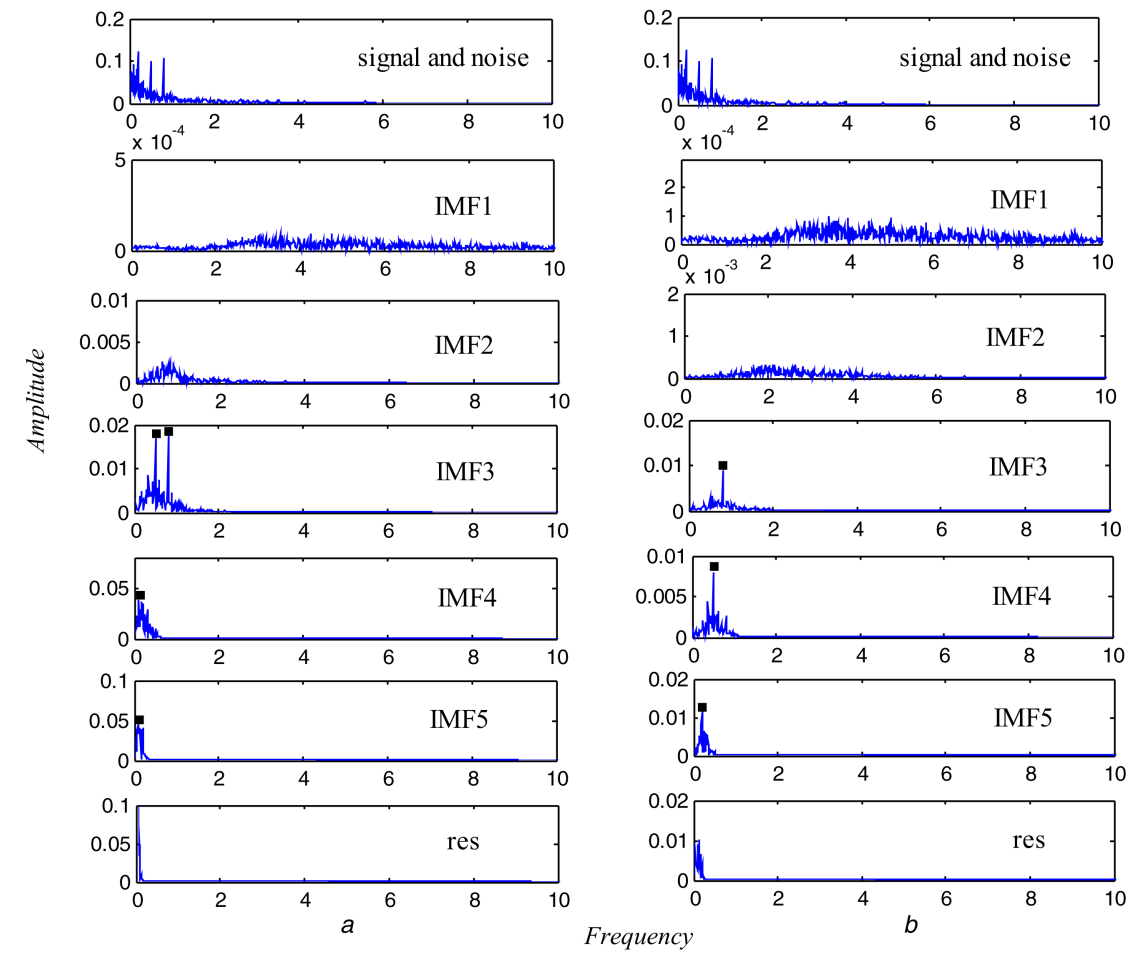

Fig. 10 Frequency spectrums of the noisy three-frequency signals are decomposed by EMD after adaptive BSR or adaptive PSR at 0.8 Hz, when the noise intensity is set as 2

(a) Frequency spectrum of the noisy three-frequency signal is decomposed by EMD after adaptive BSR at $0.8 \mathrm{~Hz}$ : optimal output with $a=0.35$ and $b=1.848$. In IMF3, the highest points are located at 0.5 and $0.8 \mathrm{~Hz}$. In IMF4 and IMF5, the highest point is located at $0.2 \mathrm{~Hz}$, (b) Frequency spectrum of the noisy three-frequency signal is decomposed by EMD after adaptive PSR at $0.8 \mathrm{~Hz}$ : optimal output with $a=1.995$ and $b=1.97$. In IMF3, the highest point is located at $0.8 \mathrm{~Hz}$. In IMF4, the highest point is located at $0.5 \mathrm{~Hz}$. In IMF5, the highest point is located at $0.2 \mathrm{~Hz}$

it is decomposed failure because of the mode mixing phenomenon. Fig. $10 b$ shows the frequency spectrum of the combination of adaptive PSR and EMD which is optimised at $0.8 \mathrm{~Hz}$. In Fig. $10 \mathrm{~b}$, $0.8,0.5$ and $0.2 \mathrm{~Hz}$ appear in IMF3, IMF4 and IMF5, respectively.
Similar to the fact in Figs. $6 b$ and $7 b$, it is also a successful decomposition.

Comparing figures treated by the combination of adaptive BSR and EMD with figures handled by the combination of adaptive 
PSR and EMD, we clearly find the complicated signal under coloured noise background by the latter method has a better decomposition effect. Furthermore, we convincingly prove the validity of this method by statistical analysis and different noisy intensities.

\section{Conclusion}

In this paper, we focus on the decomposition of a multi-frequency signal in the coloured noise background. The noisy signal cannot be successfully decomposed by EMD directly. Furthermore, the results are even unsatisfactory through the cooperation of the adaptive BSR and the EMD method. We solve the problem by the combination of adaptive PSR with EMD and get the following advantages:

(i) The research of the signal under coloured noise background is more closer to the engineering practice.

(ii) Neither the original signal nor the noisy signal is decomposed unsuccessfully by EMD directly. Moreover, this noisy signal even cannot be decomposed satisfactory by the combination of the adaptive BSR and the EMD method. However, the method we put forward has effectively solved this problem. In addition, the method we proposed might be useful in other similar signal processing problems.

Although this proposed method has good effect in processing multi-frequency signal, it still has some weakness when the noise frequency is closer to the signal frequency or signal frequencies closer to each other, or the special case, such as noise intensity is too strong. Concerning these issues, some other techniques need to be introduced to solve the problem. Moreover, we only study the low-frequency signals to verify the effectiveness of proposed method here. If we need to process the high-frequency signals, we should introduce other methods together with this proposed method, such as frequency-shifted and rescaling SR, the twice sampling SR, and the multiscale noise tuning SR, etc. This is our main research content in the future and we predict the proposed method is also applicable to high-frequency signals.

\section{Acknowledgments}

The authors acknowledge financial supports by the National Natural Science Foundation of China (grant nos. 11672325 and 51575511), Shanghai Maritime University Graduate Student Innovation (grant no. 2015ycx073) and Zhejiang Province Department of Education (201534139). The authors were also very grateful to the anonymous reviewers for their useful comments and advice, which are vital for improving the quality of this paper.

\section{References}

[1] Huang, N.E., Shen, Z., Long, S.R., et al.: 'The empirical mode decomposition and the Hilbert spectrum for nonlinear and non-stationary time series analysis', Proc. R. Soc. Lond. A, 1998, 454, pp. 903-995

[2] Wang, J., Shang, P.J., Xia, J.A., et al.: 'EMD based refined composite multiscale entropy analysis of complex signals', Physica A, 2015, 421, pp. 583-593

[3] Saidi, L., Ali, J.B., Fnaiech, F.: 'Bi-spectrum based-EMD applied to the nonstationary vibration signals for bearing faults diagnosis', ISA Trans., 2014, 53, pp. $1650-1660$

[4] Lv, Y., Yuan, R., Song, G.B.: 'Multivariate empirical mode decomposition and its application to fault diagnosis of rolling bearing', Mech. Syst. Signal Process., 2016, 81, pp. 219-234

[5] Feng, Z.P., Liang, M., Zhang, Y., et al.: 'Fault diagnosis for wind turbine planetary gearboxes via demodulation analysis based on ensemble empirical mode decomposition and energy separation', Renew. Energy, 2012, 47, pp. $112-126$

[6] Chen, X.H., Cheng, G., Shan, X.L., et al.: 'Research of weak fault feature information extraction of planetary gear based on ensemble empirical mode decomposition and adaptive stochastic resonance', Measurement, 2015, 73, pp. $55-67$

[7] Shi, P.M., An, S.J., Li, P., et al.: 'Signal feature extraction based on cascaded multi-stable stochastic resonance denoising and EMD method', Measurement, 2016, 90, pp. 318-328

[8] Benzi, R., Sutera, A., Vulpiana, A.: 'The mechanism of stochastic resonance', J. Phys. A, Math. Gen., 1981, 14, pp. 453-457

[9] Li, J., Chen, X., He, Z.: 'Adaptive stochastic resonance method for impact signal detection based on sliding window', Mech. Syst. Signal Process., 2013, 36, pp. 240-255

[10] Qin, Y., Tao, Y., He, Y., et al.: 'Adaptive bistable stochastic resonance and its application in mechanical fault feature extraction', J. Sound Vib., 2014, 333, pp. 7386-7400

[11] Zhou, B.C., Xu, W.: 'Stochastic resonance in an asymmetric bistable system driven by mixed periodic force and noises', Acta Phys. Sin-ch. ed, 2007, 56, pp. 5623-5628

[12] Long, F., Guo, W., Mei, D.C.: 'Stochastic resonance induced by bounded noise and periodic signal in an asymmetric bistable system', Physica A, 2012 391, pp. 5305-5310

[13] Nicolis, C.: 'Stochastic resonance in multistable systems: the role of intermediate states', Phys. Rev. E, 2010, 82, p. 011139

[14] Saikia, S.: 'The role of damping on stochastic resonance in a periodic potential', Physica A, 2014, 416, pp. 411-420

[15] Liu, K.H., Jin, Y.F.: 'Stochastic resonance in periodic potentials driven by colored noise', Physica A, 2013, 392, pp. 5283-5288

[16] Yang, J.H., Liu, X.B.: 'Sequential vibrational resonance in multistable systems', 2011, preprint 1106.3431

[17] Liu, X.L., Liu, H.G., Yang, J.H., et al.: 'Improving the bearing fault diagnosis efficiency by the adaptive stochastic resonance in a new nonlinear system', Mech. Syst. Signal Process., 2017, 96, pp. 58-76

[18] Wang, C.Y., Gao, Y., Song, Y.M., et al.: 'Stochastic systems with crosscorrelated Gaussian white noises', Chinese Phys. B, 2010, 19, p. 116401

[19] Prigogine, I., Rice, S.A.: 'Colored noise in dynamical systems', Adv. Chem Phys., 2007, 48, p. 239

[20] Hanggi, P., Moss, F., Mcclintock, P.V.E.: 'Bistability driven by colored noise: theory and experiment', Phys. Rev. A, 1985, 32, pp. 695-698

[21] Jung, P., Hanggi, P.: 'Dynamical systems: a unified colored-noise approximation', Phys. Rev. A, 1987, 35, pp. 4464-4466

[22] Hanggi, P., Jung, P., Zerbe, C., et al.: 'Can colored noise improve stochastic resonance', J. Stat. Phys., 1993, 70, pp. 25-47

[23] Beato, V., Sendiña, N.I., Gerdes, I., et al.: 'Coherence resonance in a chemical excitable system driven by colored noise', Philos. T. Roy. Soc. A, 2008, 366, pp. 381-395

[24] BarajasSolano, D.A., Tartakovsky, A.M.: 'Probabilistic density function method for nonlinear dynamical systems driven by colored noise', Phys. Rev. $E$, 2016, 93, p. 052121

[25] Yang, B., Zhang, X., Zhang, L., et al.: 'Collective behavior of globally coupled Langevin equations with colored noise in the presence of stochastic resonance', Phys. Rev. E, 2016, 94, p. 022119

[26] Fang, K., Hu, S.Q., Liu, Q.W., et al.: 'Effects of the colored noise on the resonance at the subharmonic frequency in bistable systems', Chinese $J$. Phys., 2017, 55, pp. 989-995

[27] Xu, B., Li, J., Duan, F., et al.: 'Effects of colored noise on multi-frequency signal processing via stochastic resonance with tuning system parameters', Chaos Soliton. Fract., 2003, 16, pp. 93-106

[28] Ma, J., Xiao, T.J., Hou, Z.H., et al.: 'Coherence resonance induced by colored noise near Hopf bifurcation', Chaos, 2008, 18, p. 043116

[29] Yang, Y.F., Wu, Y.F.: 'Applications of empirical mode decomposition in vibration analysis' (National Defense Industry Press, Beijing, China, 2013, 1st edn.), pp. 19-20

[30] Xu, B., Duan, F., Bao, R., et al.: 'Stochastic resonance with tuning system parameters: the application of bistable systems in signal processing', Chaos Soliton. Fract., 2002, 13, pp. 633-644

[31] Gammaitoni, L., Hanggi, P., Jung, P., et al.: 'Stochastic resonance', Rev. Mod. Phys., 1998, 70, pp. 223-287

[32] Mcnamara, B., Wiesenfeld K., 'Theory of stochastic resonance', Phys. Rev. A, 1989, 39, pp. 4854-4869

[33] Lu, S.L. He, Q.B., Zhang, H.B. et al.: 'Enhanced rotating machine fault diagnosis based on time-delayed feedback stochastic resonance', J. Vib. Acoust., 2015, 137, p. 051008

[34] Marini, F., Walczak, B.: 'Particle swarm optimization (PSO). A tutorial', Chemometr. Intell. Lab., 2015, 149, pp. 153-165

[35] Guedria, N.B.: 'Improved accelerated PSO algorithm for mechanical engineering optimization problems', Appl. Soft Comput., 2016, 40, pp. 455 engin
467

[36] Tsoulos, I.G., Stavrakoudis, A.: 'Enhancing PSO methods for global optimization', Appl. Math. Comput., 2010, 216, pp. 2988-3001

[37] Ichiki, A., Tadokoro, Y.: 'Signal-to-noise ratio improvement by stochastic resonance in moments in non-dynamical systems with multiple states', Phys. Let. A, 2013, 377, pp. 185-188

[38] Hu, H.Y.: 'Applied nonlinear dynamics' (China Aeronautical Industry Press, Beijing, China, 2000, 1st edn.), pp. 68-75 